This is the final peer-reviewed accepted manuscript of:
Mirasoli M, Bonvicini F, Lovecchio N, Petrucci G, Zangheri M, Calabria D, Costantini F, Roda A,
Gallinella G, Caputo D, de Cesare G, Nascetti A. On-chip LAMP-BART reaction for viral DNA real-
time biolumiscence detection. Sensors and Actuators B: Chemical. 2018.
The final published version is available online at: $\mathbf{1 0 . 1 0 1 6 / j . s n b . 2 0 1 8 . 0 2 . 0 8 6}$

Rights / License:

The terms and conditions for the reuse of this version of the manuscript are specified in the publishing policy. For all terms of use and more information see the publisher's website. 


\title{
On-chip LAMP-BART reaction for viral DNA real-time bioluminescence detection
}

\author{
M. Mirasoli a, F. Bonvicini ${ }^{\text {b }}$, N. Lovecchio ${ }^{\text {c }}$, G. Petrucci ${ }^{\text {c }}$, M. Zangheri ${ }^{\text {a }}$, D. Calabria ${ }^{\text {a }}$, F.

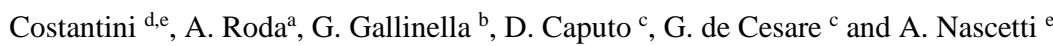

aDepartment of Chemistry “Giacomo Ciamician”, Alma Mater Studiorum - University of Bologna, via Selmi 2, 40126 Bologna, Italy

${ }^{\mathrm{b}}$ Department of Pharmacy and Biotechnology, Alma Mater Studiorum - University of Bologna, via Belmeloro 6, 40126 Bologna, Italy

'Department of Information, Electronics and Communication Engineering, University of Rome "La Sapienza", via Eudossiana 18, 00184 Rome, Italy

'Department of Chemistry, University of Rome "La Sapienza", piazzale A. Moro, 5, 00185 Rome, Italy

'Aerospace Engineering School, University of Rome "La Sapienza", via Salaria 851/881, 00138 Rome, Italy

The present paper describes the development of an integrated lab-on-chip, in which viral DNA amplification with real-time on-chip detection is carried out under constant temperature of $65^{\circ} \mathrm{C}$. The lab-on-chip is composed of a disposable $10-\mu \mathrm{L}$ polydimethylsiloxane reaction chamber which is thermally and optically coupled to a glass substrate that hosts a thin-film metallic resistive heater and thin-film amorphous silicon diodes which act as temperature and radiation sensors. A loopmediated isothermal amplification (LAMP) technique was optimized to specifically amplify parvovirus B19 DNA and coupled with Bioluminescent Assay in Real Time (BART) technology to provide real-time detection of target DNA. The experimental results demonstrate the ability of the proposed device to discriminate among different concentrations of viral DNA with an excellent agreement with standard off-chip methods.

Keywords:

Lab-on-chip; Loop-mediated isothermal amplification LAMP; Bioluminescent Assay in Real Time BART; viral nucleic acids detection; amorphous silicon sensors; thin film technology 


\section{Introduction}

The development of miniaturized analytical devices suitable for the sensitive, specific and quantitative detection of pathology biomarkers in biological samples, suitable for point-of-care (POC) applications [1], is one of the hardest challenges in analytical chemistry. The gold standard for accurate diagnosis of infectious diseases is quantitative detection of nucleic acids. Several papers have been published, describing the development of lab-on-chip devices suitable for nucleic acids detection [2-4], and few diagnostic applications have also been reported [5-7].

Particularly relevant in this context are nucleic acid amplification techniques [8] which provide low limits of detection, rapidity, reliability of results, and, when coupled with real-time quantitative detection, reduced contamination issues.

Presently, isothermal nucleic acid amplification techniques (iNAAT) are receiving growing attention [9], as they are particularly suited for POC implementation. Indeed, they only require application of a constant temperature (typically comprised between 40 and $65^{\circ} \mathrm{C}$ ) along the whole amplification process, thus greatly simplifying the on-chip thermal control task and reducing operating power consumption. In particular, loop-mediated isothermal amplification (LAMP) is a very promising technique [10], offering high specificity, sensitivity, rapidity and simple detection of amplification products [11]. High specificity of LAMP reactions, claimed as a result of their intrinsic mechanism, permits amplicon detection by means of non-sequence-specific measurements, such as turbidity, fluorescence or electrochemical measurement [10, 12]. Nevertheless, recent reports have evidenced that LAMP can lead to non-specific products, especially when experimental conditions are not carefully controlled [13].

For this reason, lab-on-chip devices for LAMP should be designed to enable accurate and continuous control of reaction conditions along the whole experiment. In addition, since real-time PCR detection of targets has become a standard method in the field of molecular diagnostics, real time on-chip monitoring of amplification products shall also be implemented, to obtain, through a smart integration approach, a self-standing miniaturized device for POC applications.

In this work, human parvovirus B19 (B19V) was taken as a model virus, as it is a ubiquitous virus with a wide pathogenic potential, including life-threatening consequences [14]. A LAMP technique for the detection of B19V DNA has been developed and the experimental factors affecting its specificity and sensitivity have been evaluated.

The preliminary experiments showed that the value of the temperature is crucial for ensuring the specificity of the reaction, thus confirming that on-chip integrated real-time temperature control is a key aspect to ensure reliable results. This requirement has been achieved by developing an 
integrated chip (named LAMP-chip), which combines on the same glass substrate thin film heaters $[15,16]$ and amorphous silicon $(\mathrm{a}-\mathrm{Si}: \mathrm{H})$ temperature sensors coupled with a biocompatible, transparent polydimethylsiloxane (PDMS) microfluidic network [17].

To obtain real-time quantitative detection of the target nucleic acid and detectability levels suitable for diagnostic applications, we coupled B19V DNA LAMP with the Bioluminescent Assay in Real Time (BART), which has been previously shown to provide simple, robust and low-cost real-time monitoring of LAMP amplification in a closed-tube format, thus greatly reducing crosscontamination risks [18-20]. Bioluminescence-based detection principles are particularly suitable for lab-on-chip applications, as they provide high detectability, high specificity, low-background signal and no need for excitation sources and filters [21-23].

In order to enable on-chip detection of the BL signal, the LAMP-chip was equipped with thin-film a-Si:H photosensors, that are fabricated with the same technological processes utilized to define the a-Si:H temperature sensors [24]. a-Si:H photosensors have already shown optoelectronic performances well suited for biochemical analysis thanks to their very low dark current and high photo-sensitivity [25]. In particular, it has been demonstrated that a-Si:H photosensors are appropriate in detecting luminescent signals coming from labeled molecules [26], and that the analytical performances of a-Si:H photosensors in detecting biomolecules through BL-based measurements are comparable to those of crystalline silicon cooled charge-coupled device (CCD)based state-of-the-art laboratory imaging instrumentation [27, 28]. Additionally, the low deposition temperature (below $300^{\circ} \mathrm{C}$ ) enables the possibility to use glass, the traditional support for biochemical analysis, as substrate for the a-Si:H photosensors [29-32].

\subsection{Materials}

B19V DNA target for experiments consisted of purified nucleic acids obtained from infected cell culture supernatant. Briefly, erythroid progenitor cells generated in vitro from peripheral blood mononuclear cells were inoculated with a B19V viremic serum and, following infection, the virus released in the supernatant was processed by using the EZ1 viral nucleic acid kit on a EZ1 platform (Qiagen, Germany) [33]. B19V DNA was quantified using a qPCR assay, and the DNA amount was expressed as viral genomes [34]. Aliquots of serial 10-fold dilutions of the template were stored at $-20^{\circ} \mathrm{C}$ until application for LAMP reactions.

Primers targeting B19V were purchased by Eurofins Genomics (Germany). To prepare in-tube LAMP reactions, 10× ThermoPol reaction buffer, $100 \mathrm{mM} \mathrm{MgSO}_{4}$ and Bst DNA polymerase large fragment were purchased from New England BioLabs (MA, USA); 100 mM dATP, dCTP, dGTP, 
dTTP and $5 \mathrm{M}$ betaine solution were ordered from Sigma-Aldrich (MO, USA). LAMP-BART reagents were generously provided by ERBA Mannheim Molecular (Cambridge, UK).

\subsection{Primer design}

The central region of B19V genome (NCBI Genome Database: accession number NC_000883.2) was selected and used as target for LAMP primers' design. Two sets of four primers (outer primers [F3 and B3] and inner primers [FIP and BIP]) were automatically designed using the LAMP primer design software PrimerExplorer V4 (https://primerexplorer.jp), and a further set was manually designed using the Clone Manager 9 Professional Edition software (NC, USA), and following the general criteria of LAMP design described by Eiken Chemical Co [35]. Three primer sets were evaluated and are reported in the Supplementary Information (SI).

\subsection{LAMP reaction}

In-tube LAMP reaction was carried out using a conventional benchtop PTC-100 thermal cycler (MJ Research, USA) in a final volume of $20 \mu \mathrm{L}$ containing $1.6 \mu \mathrm{M}$ each FIP and BIP, $0.2 \mu \mathrm{M}$ each F3 and B3, $400 \mu \mathrm{M}$ each dNTPs, $1 \mathrm{M}$ betaine, $2 \mathrm{mM} \mathrm{MgSO}_{4}, 1 \times$ ThermoPol reaction buffer, $4 \mathrm{U}$ Bst DNA Polymerase, and B19V DNA in the range of $10^{0}$ to $10^{5}$ viral genomes, previously heated at $95^{\circ} \mathrm{C}$ for $5 \mathrm{~min}$ and chilled in ice for $5 \mathrm{~min}$. The optimized LAMP reaction was performed by using primer set 3 , at $65^{\circ} \mathrm{C}$ for $50 \mathrm{~min}$ and terminated by heating up to $80^{\circ} \mathrm{C}$ for $10 \mathrm{~min}$.

\subsection{LAMP-BART reaction}

The off-chip LAMP-BART reaction was carried out on an assembled instrument comprising a conventional heating block for microtiter plates and an imaging system constituted of a MZ-2PRO CCD camera (MagZero, Pordenone, Italy) equipped with a thermoelectrically cooled monochrome Sony ICX285 CCD image sensor, coupled with a Computar (2/3 in. $8 \mathrm{~mm}, \mathrm{f} 1.4)$ objective (CBC AMERICA Corp., Commack, NY), and connected to a light-tight dark box to avoid interference from ambient light [36].

The reaction was prepared in a black 384-well plate in a final volume of $20 \mu \mathrm{L}$, covered with mineral oil to prevent evaporation. The lyophilized LAMP-BART master mix was reconstituted following manufacturer's instructions (ERBA Mannheim Molecular, Cambridge, UK). Primer set 3 (1.6 $\mu \mathrm{M}$ each FIP and BIP, $0.2 \mu \mathrm{M}$ each F3 and B3) were used to amplify B19V DNA dilutions in the range of $10^{0}$ to $10^{7}$ viral genomes, previously denatured at $95^{\circ} \mathrm{C}$ for $5 \mathrm{~min}$, and chilled in ice. The optimized LAMP-BART reaction was performed at $65^{\circ} \mathrm{C}$. Photon emission was acquired for all the samples throughout the amplification by integrating over 30 -s intervals, for a 40 -min total 
time. Images were elaborated employing the WinLight32 software version 2.91 (Berthold Technologies GmbH\&Co. KG, Bad Wildbad, Germany). For each image the area corresponding to each well was selected and the BL signal integrated; the blank was measured in the area corresponding to an empty well and subtracted from each well signal. For quantification of B19V DNA, a concept of time to peak $\left(\mathrm{t}_{\max }\right)$, defined as the time to maximum light output recorded during the reaction, was applied. The $t_{\max }$ is directly correlated with target DNA copy number [18].

\subsection{On-chip LAMP-BART reaction}

The LAMP-BART reaction was assembled and mixed off-chip before the real-time analysis in the integrated LAMP-chip equipped with a PDMS microfluidic module. The microchannel was filled up with $10 \mu \mathrm{L}$ of LAMP-BART mixture, taking care to avoid the presence of air bubbles in the reaction chamber, and tightly sealed with adhesive film to prevent evaporation from sample inlet/outlet. The amplification was carried out by heating up the micro-chamber at $65^{\circ} \mathrm{C}$.

The feasibility of the microdevice was demonstrated with samples containing $10^{5}$ or $10^{7} \mathrm{~B} 19 \mathrm{~V}$ viral genomes; each experiment was repeated 3 times to ensure the robustness and reproducibility of the developed LAMP-chip.

The LAMP-chip was placed into a light-shield box to prevent interference from ambient light. Photon emission was measured by the on-chip a-Si:H photodiodes which absorb the impinging light and convert it in electron and hole pairs. The photocurrent is then acquired by a low-noise read-out system [37]. The readout electronics relies on a charge sensitive preamplifier (DDC118 from Texas Instruments) that integrates the sensor current giving as output a proportional voltage. The whole electronic board is controlled by a microcontroller (PIC18F4550 from Microchip) that also provides the USB connection to a PC for setting of the measurement parameters (acquisition time, integration time), data storage and result visualization [38]. At each measurement, a preliminary measurement of the sensor dark current has been performed to calculate the photocurrent.

\subsection{Qualitative analysis of LAMP products}

Following LAMP and LAMP-BART reactions, a $2-\mu \mathrm{L}$ aliquot of amplification product was resolved in a $2 \%$ standard agarose gel electrophoresis, and the ladder-like pattern generated from the multiple repeat of the targeted B19V sequence was visualized over UV light (VersaDoc Image System Model 1000, BioRad, CA, USA). In addition, to confirm the DNA identity and size of the amplified products, a standard Southern blot analysis was performed by using a full-length digoxigenin-labeled B19V DNA probe for hybridization and colorimetric detection to reveal B19V specific hybrids [39]. 


\subsection{Design and fabrication of the LAMP-chip}

The integrated LAMP-chip combines on the same $5 \times 5-\mathrm{cm}^{2}$ glass substrate thin-film metal and amorphous silicon (a-Si:H)/amorphous silicon carbide (a-SiC:H) stacked structures. The thin-film metal layers are deposited on one side of the glass substrate, while the a-Si:H/a-SiC:H devices are grown on the other side. In particular, the thin metal film acts as resistive heating source, the a-Si:H devices act as both temperature sensors (to monitor the temperature distribution on the active area of the heater) and photosensors (for the on-chip detection of the BART reaction).

2.7.1 Thin film heaters. The thin film heater is a stack of $\mathrm{Cr} / \mathrm{Al} / \mathrm{Cr}$ films, deposited by vacuum evaporation (in a Balzers 510 evaporation system) and patterned by photolitographic process. Its geometry has been designed, by using COMSOL Multiphysiscs, to ensure a uniform temperature distribution over the entire area where the LAMP-BART reaction and DNA amplification occur. Material selection and layers thicknesses have been also chosen aiming to achieve a low resistance that could be driven with a USB supply (at maximum $5 \mathrm{~V}$ and $500 \mathrm{~mA}$ ). A layer of conductive transparent material, Indium Thin Oxide (ITO) [40] has been first deposited on the glass substrate and then covered with an insulation plane made of SU-8 3005.The ITO layer acts both as electromagnetic interferences (EMI) shield as well as leakage guard plane for the on-chip sensors [3]. The heater has been then fabricated by vacuum evaporation (in a Balzers 510 evaporation system) and subsequently patterned by conventional photolitographic process.

The heater performances have been characterized by measuring the temperature distribution on the opposite glass side (namely the side hosting the a-Si:H/a-SiC:H sensors) by using a FLIR A325 thermo camera. Data have been evaluated through the software of the thermo camera.

2.7.2 Amorphous silicon sensors. The a-Si:H/a-SiC:H structures are n-type/intrinsic/p-type junctions. The amorphous silicon layers were deposited in a high-vacuum system by Plasma Enhanced Chemical Vapor Deposition (PECVD) at $13.56 \mathrm{MHz}$ and patterned using standard microelectronic fabrication technologies (see SI).

Sensors' positions, schematically sketched in Fig. 1, guarantees the on-chip and real time detection of the BL reaction occurring inside the microfluidic channel as well as the real-time monitoring of the temperature distribution [41].

A cross section of the complete structure is depicted in Fig. 1b. The ITO and SU-8 layers below the glass substrate represent the ground plane structure that avoids interferences of the heater driving currents with the photosensor signals [3]. The bottom contact of both sensors is a 
$30 \mathrm{~nm} / 150 \mathrm{~nm} / 30 \mathrm{~nm}$-thick stack of $\mathrm{Cr} / \mathrm{Al} / \mathrm{Cr}$ layer, while the top contact, exposed to the impinging light, is a metal layer for the temperature sensor and a grid electrode for the photosensor [42]. In this way, the light can be absorbed by the photosensors, while it is reflected by the metal contact of the temperature sensors.

The sequence of the fabrication steps and the selection of the appropriate coating materials was optimized in order to ensure the successful integration of the heater and the a-Si:H diodes on the same glass avoiding damages or incorrect operation of the previously fabricated structures. The entire fabrication process is reported in the SI.

\subsection{Microfluidic network}

Polydimethylsiloxane (PDMS) Sylgard 184 pre-polymer and curing agents were purchased from Dow Corning (Midland, MI). By combining the two ingredients in specific ratios and curing them at specified conditions, PDMS of different properties can be obtained [43]. The soft lithography fabrication process includes the fabrication of a master, the pouring of the PDMS polymer and the curing at a specified temperature for the complete polymerization. The developed network, shown in Fig. 1, includes two linear channels (blue rectangles). The dimensions of each channel (18.1 mm x $2.5 \mathrm{~mm} \times 0.195 \mathrm{~mm}$ ) have been set in order to obtain the right volume for the BART implementation (about $10 \mu \mathrm{L}$ ). The positioning of the microfluidic network over the glass substrate guarantees a proper alignment with the photosensors (green squares) and the temperature sensors (red squares).

In our process, first, a master was obtained by bonding a stack of three layers of $65 \mu \mathrm{m}$-thick kapton tape over a silicon substrate. Length and width of the tape reproduce exactly the channel dimensions. A mixture of silicone pre-polymer and curing agent in the ratio of 10:1 was prepared and poured over the master. The thickness of the PDMS mold was about $3 \mathrm{~mm}$. The PDMS was cured in oven at $60^{\circ} \mathrm{C}$ for $150 \mathrm{~min}$. Finally, channel inlet and outlet holes were made with a puncher (0.021 in. ID $\times 0.028$ in. OD $\times 1.5$ in. long purchased from Syneo LLC, Angleton, TX, USA). The PDMS mold was then bonded to a cleaned glass substrate [44]. 


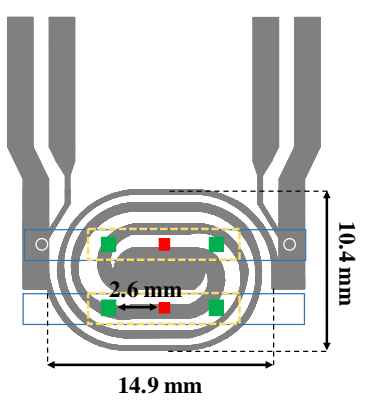

a)

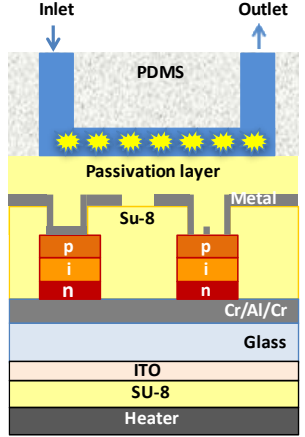

b)

Fig. 1 a) Top view of the main parts of the LAMP-chip. Two microfluidic channels (light-blue rectangles), showing the alignment with the a-Si:H photosensors (green squares with $1.1 \mathrm{~mm}$ side) and temperature sensors (red squares with $0.8 \mathrm{~mm}$ side). The gray lines represent the profile of thin film heater, the white circles are the holes for the inlet and the outlet of each channel, the dashed yellow rectangles represent the useful area of the microfluidic channel. b) Cross section of the whole device. The p-i-n structures are the a-Si:H sensors. The one on the left, with a continuous metal contact is the temperature sensor, while the one on the right, with a grid top electrode is the photosensor.

\section{Results and Discussions}

\subsection{LAMP-BART optimization for B19V DNA quantitative detection}

3.1.1 LAMP reaction for B19V DNA. Appropriate design of the primer set plays a pivotal role in the success of LAMP reaction. The technique utilizes four primers targeting six regions within a small segment ( $<200 \mathrm{bp}$ ) of a target DNA, so that the specificity is supposed to be extremely high [35]. However, due to the high concentrations of primers used in the reaction compared to traditional PCR methods (>3-fold) together with the numerous sets of primers employed, the generation of primer dimers and non-specific amplification products has been observed, mainly in the absence of strict control of experimental conditions [13].

In this study, three sets of four primers were designed to target the central region of B19V genome (Table $1, \mathrm{SI}$ ), and they were used to amplify a sample containing $10^{3}$ copies of B19V DNA, in a preliminary subset of experiments performed at $65^{\circ} \mathrm{C}$ for $60 \mathrm{~min}$. LAMP products were analyzed in a $2 \%$ standard agarose. As the product of LAMP amplification is a mixture of stem-loop DNAs, a pattern of several bands, which position is typical for each set of primers for a given target, is expected on the gel. Even if all sets complied with the requirements of LAMP primer design [22], primer set 1 failed to amplify B19V DNA, confirming that the subtle definition of the experimental parameters, including primer selection, is necessary for a successful LAMP reaction. Primer sets 2 
and 3 led to the generation of the expected ladder-like pattern of products, however, bands obtained with primer set 2 were weaker than 3 , thus primer set 3 was employed for the subsequent reactions. This primer set has been previously shown to be suitable for B19V DNA LAMP amplification [45].

3.1.2 Study of the effects of temperature and incubation time on LAMP reaction. At a suitable temperature, the Bst DNA polymerase used in LAMP reactions has polymerase activity and strand displacement ability leading to the separation of the non-template strand from the template DNA without the thermal cycles of the PCR process. To guarantee the choice of the correct incubation temperature ensuring both sensitivity and specificity, different isothermal conditions were assayed, $57^{\circ} \mathrm{C}, 60^{\circ} \mathrm{C}, 63^{\circ} \mathrm{C}, 65^{\circ} \mathrm{C}, 68^{\circ} \mathrm{C}$ and $70^{\circ} \mathrm{C}$, using $10^{3} \mathrm{~B} 19 \mathrm{~V}$ viral genomes as template and a standard PCR-tube format. Subsequently, LAMP products were analyzed by gel electrophoresis (Figure 2a). Specific and efficient B19V amplification occurred only at $63^{\circ} \mathrm{C}$ and $65^{\circ} \mathrm{C}$, while at temperatures higher than $65^{\circ} \mathrm{C}$ no amplification was observed, presumably because of Bst DNA polymerase inactivation or primers inefficient annealing to the target. At $57^{\circ} \mathrm{C}$ and $60^{\circ} \mathrm{C}$ a non-specific reaction occurred leading to the generation of mispriming or primer dimers with a ladder-like pattern different from the specific LAMP product. Indeed, the shortest band in the specific LAMP product is expected to be $147 \mathrm{bp}$ in length, that is the distance between FIP and BIP primers on B19V genome (Fig. S1, SI). The shorter fragments observed in LAMP reactions performed at temperatures $\leq 60^{\circ} \mathrm{C}$ demonstrate non-specific amplification, despite the presence of cellular exogenous DNA in the sample and of betaine, which have been previously reported to improve amplification specificity [22]. Since the amount of products obtained by LAMP reaction at $65^{\circ} \mathrm{C}$ was slightly more abundant, as compared with that obtained at $63^{\circ} \mathrm{C}, 65^{\circ} \mathrm{C}$ temperature was set for all subsequent experiments.

To evaluate the limit of detection of the LAMP, different amounts of B19V DNA target, from $10^{0}$ to $10^{5}$ copies/reaction, were amplified at $65^{\circ} \mathrm{C}$ for $60 \mathrm{~min}$ in a conventional laboratory equipment. As reported in Fig. 2d, specific LAMP products were obtained at target DNA amounts $\geq 10^{2}$ copies/reaction, while non-specific products with a band pattern similar to that previously observed for inappropriate temperature conditions were obtained at the lowest target amounts $\left(\leq 10^{1}\right.$ copies/reaction) and in the absence of template (NTC). Such results were also confirmed by Southern blot hybridization assay (Fig. S2, SI).

On the other hand, it was observed that by conducting the amplification reactions at $65^{\circ} \mathrm{C}$ for shorter times (40 and $50 \mathrm{~min}$ ), no nonspecific amplicon was detected at low or zero target amounts (Fig. 2b-d). In particular, while after 40 min of incubation only the highest DNA amounts $\left(>10^{3}\right.$ 
copies) were amplified, after 50 min B19V targets $\geq 10^{2}$ copies/reaction were specifically amplified, as confirmed by Southern blot hybridization assay.

The above reported results can be explained considering that in iNAAT approaches, as well as in PCR-based methods, the amount of non-specific products increases as the amount of the target input DNA decreases, due to the competition for reaction components during the amplification process. It has indeed been previously reported that non-specific amplification can take place in the absence (or in the presence of low amounts) of target DNA, which is ascribable to the formation of LAMP equivalents of primer-dimers that can be detected by gel electrophoresis [13]. In particular, non-specific amplification of negative controls and of low abundance targets is normally a late event throughout the reaction, thus non-specific amplification and primer-dimer formation can generate and accumulate later in the reaction. Considering that both the number of primers and their concentrations in LAMP reaction is higher than in conventional PCR, the production of nonspecific products can occur, and should be considered as a limiting factor in the applicability of LAMP methodology [13]. However, as a result of a careful and detailed optimization, the developed LAMP reaction for B19V DNA, performed at $65^{\circ} \mathrm{C}$ for $50 \mathrm{~min}$, ensured the specific detection of $\mathrm{B} 19 \mathrm{~V}$ DNA with a limit of detection $\left(10^{2}\right.$ copies/reaction) suitable for clinical applications.

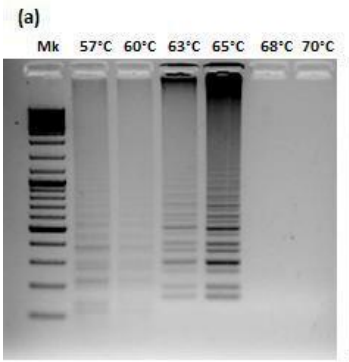

(c)

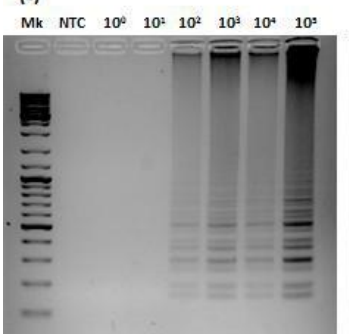

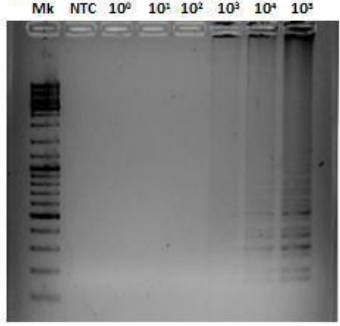

(d)
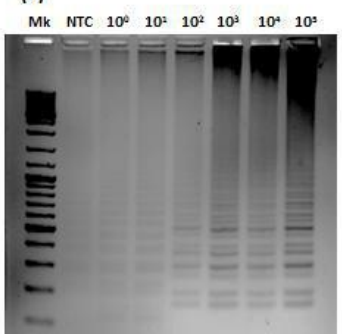

Fig. 2. Electrophoresis analysis of LAMP products obtained (a) at the indicated temperatures with a sample containing $10^{3}$ copies of B19V DNA target, and following different incubation times at $65^{\circ} \mathrm{C}: 40 \mathrm{~min}(\mathrm{~b}), 50 \mathrm{~min}(\mathrm{c})$ and $60 \mathrm{~min}$ (d) with 10-fold dilutions of B19V DNA target. Mk, 100 bps DNA ladder; NTC, no template control. 
3.1.3 LAMP-BART reaction for B19V DNA. The LAMP-BART reaction was explored to obtain the real-time detection and the quantitative analysis of B19V DNA. The technology relies on the enzymatic conversion of inorganic pyrophosphate, produced stoichiometrically during nucleic acid synthesis, into ATP (by ATP sulfurylase in the presence of adenosine-5'-O-phosphosulphate), which is then detected employing a thermostable firefly luciferase yielding a bioluminescence (BL) emission [18].

For these experiments, primer set 3 was used in a LAMP-BART reaction mixture. To check the effects of temperature on the LAMP-BART reaction, a sample containing $10^{3}$ copies of B19V DNA was amplified at $57^{\circ} \mathrm{C}, 60^{\circ} \mathrm{C}, 63^{\circ} \mathrm{C}, 65^{\circ} \mathrm{C}, 68^{\circ} \mathrm{C}$ and $70^{\circ} \mathrm{C}$ employing a closed PCR-tube configuration.

End-point analysis of products on agarose gel was carried out confirming that $65^{\circ} \mathrm{C}$ is the optimal temperature for correct B19V amplification, as previously obtained in LAMP experiments. Once again, the strict control of the temperature is crucial to avoid false positive results occurring at temperature lower than $63^{\circ} \mathrm{C}$ and false negative results at temperature higher than $65^{\circ} \mathrm{C}$ (Fig. 3 , Fig $\underline{\mathrm{S} 1})$.

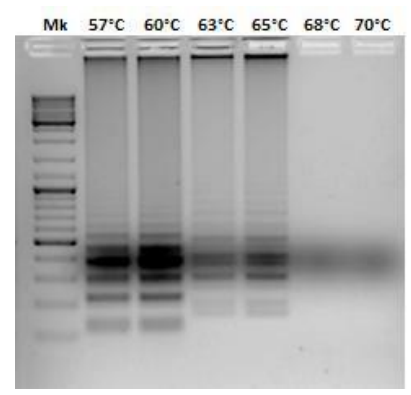

Fig. 3 Electrophoresis analysis of LAMP-BART products obtained at the indicated temperatures with a sample containing $10^{3}$ copies of B19V DNA target, and employing a closed PCR-tube configuration. Strong bands $\underline{\underline{S} \text { mear }}$ in all lanes correspond to luciferin. Mk, 100 bps DNA ladder.

Having established the optimal temperature for LAMP procedure, different amounts of B19V DNA were amplified in a black 384-well plate and light emission was recorded during the amplification by BL imaging employing a thermoelectrically-cooled back-illuminated ultrasensitive CCD camera. Data light output were plotted versus the incubation time and analyzed (Fig. 4a).

LAMP-BART kinetic curve of a positive sample consists in a distinctive "flash" (characterized by its $t_{\max }$, time to peak value) that is produced in real time when DNA amplification goes into 
exponential phase. The time taken to reach this peak of light emission is inversely proportional to the amount of target analyte. Hence, LAMP-BART technology allows for a closed tube real time quantitative evaluation of DNA in the sample and a calibration curve is generated plotting the $t_{\max }$, versus target DNA amount (Fig. 4b). A good correlation between the starting B19V DNA and $t_{\max }$, was retained, with a limit of detection of 10 copies/reaction. Compared to LAMP system, the reaction time of LAMP-BART was shorter and within 20 min all B19V DNA targets proved to be correctly amplified. Even in this experimental set-up, non-specific amplification induced by primer dimers could occur at temperature lower than $63^{\circ} \mathrm{C}$ in presence of B19V DNA and at later reaction $\underline{\text { time, even at the right temperature, in the absence of target. In the first event, the light peak }}$ emission becomes independent of B19V amount, affecting $t_{\max }$ value reproducibility, as primer dimer formation is unpredictable. However, all drawbacks are easily overcome with the strict temperature control and by stopping the LAMP-BART at $20 \mathrm{~min}$.

It must be noted that, even in this experimental set up, primer dimer formation oceurred at later time in the absence of target. Nevertheless, careful temperature control could ensure high reproducibility of the $t_{\max }$, values, thus primer dimer amplification could be easily distinguished from B19V specific LAMP amplification, simply stopping the LAMP BART at 20 min.
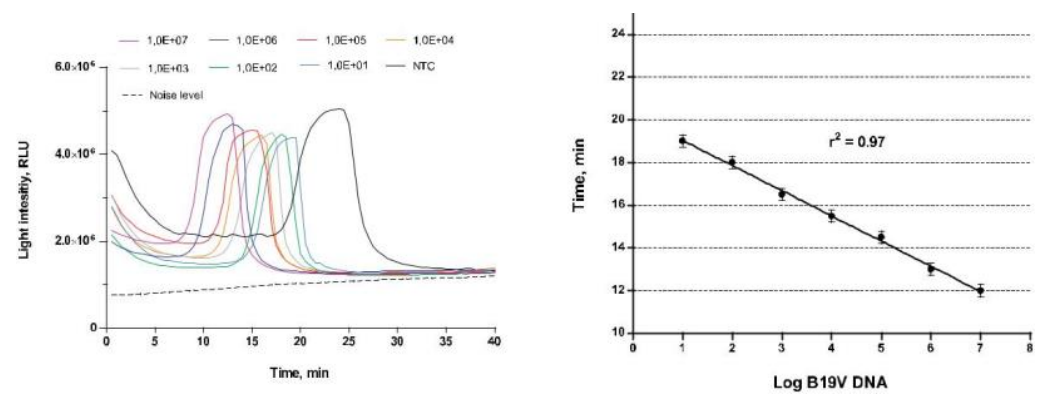

Fig. 4 LAMP-BART light intensities recorded over $40 \mathrm{~min}$ of incubation at $65^{\circ} \mathrm{C}$ for 10 -fold dilutions of B19V DNA ranging from $10^{1}$ to $10^{7}$ copies/reaction and the no template control (NTC) (a); semilogarithmic plot of the time-to-peak versus B19V DNA amounts (b).

\subsection{LAMP-chip}

3.2.1 Thin film heaters characterization. Following simulation results and previously published work [3], we found that a double concentric spiral geometry (see gray geometry in Fig. 1) ensures the required temperature uniformity over the entire reaction site [16], while a three-metal stack 
layer of $\mathrm{Cr} / \mathrm{Al} / \mathrm{Cr}$ with thicknesses equal to $100 \mathrm{~nm} / 600 \mathrm{~nm} / 100 \mathrm{~nm}$, respectively, provides a heater resistance as low as $11 \Omega$. Fig. 5a reports (in color scale) the computer simulated temperature distribution on the glass side hosting the a-Si:H/a-SiC:H sensors, when a voltage equal to $2.57 \mathrm{~V}$ is applied to the heater, calculated with COMSOL Multiphyscs. The average temperature is in the area occupied by the reaction chambers (shown as white rectangles) is $65.4^{\circ} \mathrm{C} \pm 1^{\circ} \mathrm{C}$.

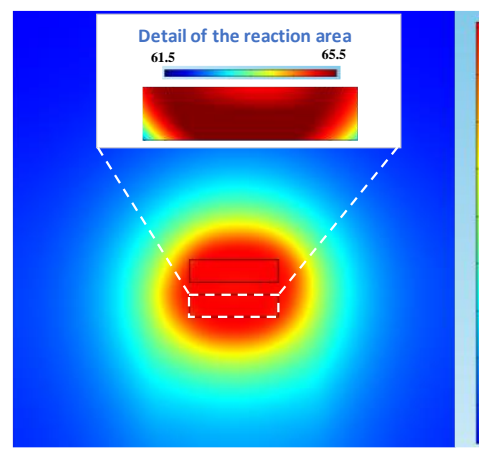

(a)

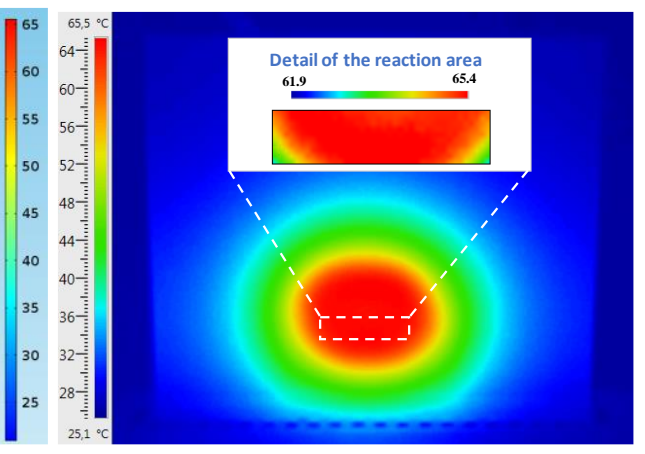

(b)

Fig. 5 (a) Finite element simulation results of the temperature distribution, achieved on the glass side hosting the a$\mathrm{Si}: \mathrm{H} / \mathrm{a}-\mathrm{SiC}: \mathrm{H}$ sensors, when a voltage equal to $2.57 \mathrm{~V}$ is applied to the heater; (b) temperature distribution measured with a thermal imager on the glass side hosting the a-Si:H/a-SiC:H sensors when a voltage equal to $2.9 \mathrm{~V}$ is applied to the metal pads. The insets in both figures show the detail of the useful area of the microfluidic channel where the sensors are located as described in Fig. 1.

Fig. 5b reports an image, in stationary condition, of the temperature distribution over the entire glass substrate $\left(5 \times 5 \mathrm{~cm}^{2}\right)$ measured using a FLIR A325 thermo camera on a fabricated device when a voltage equal to $2.9 \mathrm{~V}$ is applied to the metal pads. A quite good agreement between simulations and experimental results is achieved with a $64.9 \pm 1.0^{\circ} \mathrm{C}$ average temperature over the area above the sensors where the reaction chamber must be positioned.

3.2.2 Photosensors characterization. Energy gaps and deposition times of the different layers have been designed, using a numerical simulator [46], in order to maximize the signal-to-noise ratio of the photosensor. From the simulation results, we found that an i-layer thickness of $400 \mathrm{~nm}$ minimizes the dark current and gives a spectral response that matches quite well the emission spectrum of the BART reaction.

The amorphous silicon layers were deposited by PECVD (Table 1 in SI). The deposition time of the intrinsic layer has been set taking into account the simulation results and the film growth rate. 
After fabrication, the a-Si:H photosensors were characterized measuring their current-voltage curves in dark conditions and their quantum efficiency response. We have found that the dark current at room temperature is around $10^{-10} \mathrm{~A} / \mathrm{cm}^{2}$ at reverse bias voltage below $250 \mathrm{mV}$, independently on the photosensor area. The dark current increases exponentially with temperature [41], and reaches $30 \mathrm{pA}$ at $65^{\circ} \mathrm{C}$, the optimal temperature for the BART reaction, when the diode is reverse biased at $200 \mathrm{mV}$. Taking into account that the measurements have been performed with a signal bandwidth of $1 \mathrm{~Hz}$, the corresponding dark current noise is $3 \mathrm{fA}$, which is lower than the experimental noise introduced by the experimental set-up (around $10 \mathrm{fA}$ ) and well below the photocurrent signals detected in our previous experiments based on a-Si:H photosensors.

From the quantum efficiency curves, we derived that the responsivity, $\mathrm{R}$, is around to $320 \mathrm{~mA} \mathrm{~W}^{-1}$ at $560 \mathrm{~nm}$, which is the peak emission wavelength of the BART reaction. This value, combined with the very low dark current of the a-Si:H photodiodes, ensures a very high signal-to-noise ratio and thus very low limits of detection.

3.2.3 Temperature sensor characterization and thermal control. As outlined in the previous section, the n-type/intrinsic/p-type a-Si:H stacked structures act also as temperature sensors. In this working operation, the a-Si:H diode is biased at a constant current and the voltage drop across is proportional to its temperature, as already reported [28]. Measuring the drop voltage across the diode as a function of temperature in the range $30-100^{\circ} \mathrm{C}$, we found that the diode thermal sensitivity, is around $3.2 \mathrm{mV} /{ }^{\circ} \mathrm{C}$, which is higher than the thermal sensitivity of crystalline diodes. An electronic circuit described in detail elsewhere [47] has been used to perform the temperature control through a Proportional-Integral-Derivative (PID) algorithm. Comparing the actual diode voltage with that corresponding to the set point temperature the electronic board drives the thin film heater until the set point is reached.

\subsection{LAMP-BART reaction on the LAMP-chip}

Having demonstrated the applicability of the LAMP-BART reaction to the real-time detection of B19V DNA on a 384-well format, the technique was implemented on the LAMP-chip in which all the elements for accurate temperature control and real-time BL detection are integrated in a single support, employing compatible processes.

As a proof of concept sample containing $10^{7}$ or $10^{5}$ B19V DNA copies were amplified and the photocurrent was recorded in real time. At the beginning of the measurement, the two-channel microfluidic network has been aligned with the a-Si:H sensors [48]: one channel has been filled with the solution containing all the chemicals for the BART reaction including the target DNA and 
the other channel was used as reference by filling it with the reaction mixture without DNA (blank or control signal).

The microfluidic inlet and outlets have been then sealed with adhesive foil. Subsequently, through a custom software the diode temperature has been set to $65^{\circ} \mathrm{C}$ and the PID algorithm has been launched. The biasing current of the temperature sensors has been set to $200 \mathrm{nA}$. Simultaneously, the photosensor currents have been acquired through the low-noise read-out system.

Results are shown in Fig. 6. In Fig. 6a, the blue curve refers to a sensor aligned with the channel filled with the BART reaction, the red curve refers to a sensor aligned with the control channel.

Comparing the photocurrent profiles obtained in the LAMP-chip reactions with the light output measured in the 384-well LAMP-BART experiments, some differences in the signal shapes were evidenced. In particular, the increase of the baseline signal observed during all the measurements (blank and sample) could be ascribed to partial water evaporation and absorption of LAMP-BART reagents on PDMS [49], although it was passivated with BSA prior to use. It has been indeed previously reported that changes in LAMP-BART reagents concentration have a significant impact on the shape of the BART curves, without significantly affecting the peaking time thus ensuring the correct evaluation of the DNA target $[18,20]$.

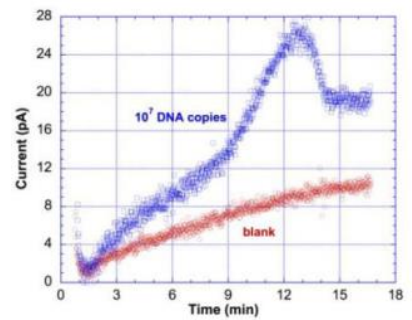

(a)

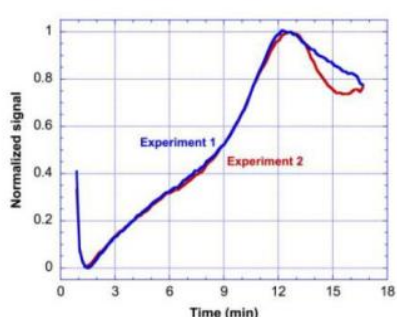

(b)

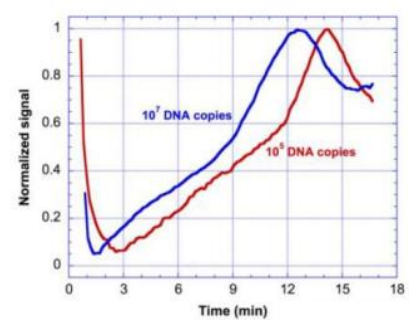

(c)

Fig. 6 Photosensor current as a function of time, acquired with the dedicated read-out electronics. (a) Signals measured when the channel has been filled with $10^{7}$ viral genomes: the red curve is the blank signal (sensor current due to background mixture signal), the blue curve refers to the total signal (sum of the currents due to temperature and to the BL emission). (b) Photocurrent signals of two photosensors monitoring the BART process in two different experiments with $10^{7} \mathrm{~B} 19 \mathrm{~V}$ DNA copies. (c) Photocurrent signals of two photosensors monitoring the BART process in two experiments when one channel contains $10^{7} \mathrm{~B} 19 \mathrm{~V}$ DNA copies and the other $10^{5} \mathrm{~B} 19 \mathrm{~V}$ DNA copies.

The photocurrent behaviour with a Gaussian-like peak, only observed when the samples contains B19 DNA, in in excellent agreement with the results achieved in the 384-well LAMP-BART experiment and the typical signal kinetics displayed for LAMP-BART assays [20]. This experiment thus demonstrates for the first time the ability to display on a single integrated chip all the functions 
required for perform a real-time LAMP reaction with on-chip optical detection. Fig. $6 \mathrm{~b}$ reports the normalized signals of two photosensors monitoring the BART reaction in a sample containing $10^{7}$ copies. We observe that the intensity peak occurs for the two photosensors at the same time, confirming the reproducibility of the whole process.

The dependence of the peaking occurrence time on the initial amount of target DNA previously reported [18-20] has been observed also in the measurements performed with our integrated lab-onchip system. The time at which the peaks occur for the two tested initial quantities of the target DNA (see Fig. 6c) are in very good agreement with those found in the off-chip experiments reported in Figure 4.

The specificity of the reactions monitored in the two above reported experiments was confirmed with agarose gel electrophoresis and southern blot analysis.

As a final consideration, even though a larger campaign of tests has to be performed to achieve an exhaustive characterization, including a complete calibration curve obtained on the LAMP-chip and analysis of real samples, these results demonstrate the suitability of the presented lab-on-chip to detect viral genomes by LAMP-BART in a really compact and miniaturized system, whose future development can bring to a portable fully integrated device fulfilling the requirements of rapidity, sensitivity, specificity, and ability to provide quantitative information for an accurate early diagnosis of viral infections in a POC environment.

\section{Conclusions}

This work presents for the first time a lab-on-chip system that integrates on a single glass substrate all the elements necessary for fine control of the temperature for iNAAT techniques and real time monitoring of the BL signal generated during nucleic acid amplification. In particular, it combines thin film microelectronic technologies and optimized LAMP-BART reactions to achieve a sensitive, compact device, which satisfies the requirements of a POC device for diagnostic applications. Thanks to the accurate temperature control, specific target DNA detection has been obtained by monitoring the time of appearance of the BL emission peak, with an excellent agreement with offchip standard methods.

\section{Acknowledgements}


Financial support was provided by Italian Ministry of Education, University and Research (MIUR) through PRIN 2010-2011 Project ARTEMIDE (ref. 20108ZSRTR) and to the MIUR through the University Research Project 2015 (prot. C26H15J3PX).

We are extremely grateful to ERBA Mannheim Molecular (Cambridge, UK) for fruitful scientific discussion and for generously providing LAMP-BART reagents. 


\section{References}

[1] W.E. Jung, J. Han, J.W. Choi, C.H. Ahn, Point-of-care testing (POCT) diagnostic systems using microfluidic lab-on-a-chip technologies, Microelectron. Eng. 132 (2015) 46-57.

[2] A.M. Foudeh, T. Fatanat Didar, T. Veres, M. Tabrizian, Microfluidic designs and techniques using lab-on-a-chip devices for pathogen detection for point-of-care diagnostics, Lab Chip. 12 (2012) 3249-66.

[3] G. Petrucci, D. Caputo, N. Lovecchio, F. Costantini, I Legnini, I. Bozzoni, A. Nascetti, G. de Cesare, Multifunctional System-on-Glass for Lab-on-Chip applications, Biosensors and Bioelectronics 93 (2017) 315-321.

[4] M.C. Giuffrida, G. Spoto, Integration of isothermal amplification methods in microfluidic devices: Recent advances, Biosensors and Bioelectronics 90 (2017) 174-186.

[5] J.J. Bella, E. J. Andersonc, W.H. Greened, J.R. Romero, M. Merchanth, R. Selvarangana, Multicenter clinical performance evaluation of BD VeritorTM System for Rapid Detection of Respiratory Syncytial, Virus. Journal of Clinical Virology 61 (2014) 113-117.

[6] M. Safavieh, M.U. Ahmed, A. Ng, M. Zourob, High-throughput real-time electrochemical monitoring of LAMP for pathogenic bacteria detection, Biosensors and Bioelectronics, 58, (2014) 101-106.

[7] M. Zangheri, M. Mirasoli, A. Nascetti, D. Caputo, F. Bonvicini, G. Gallinella, G. de Cesare, A. Roda, Microfluidic cartridge with integrated array of amorphous silicon photosensors for chemiluminescence detection of viral DNA, Sensing and Bio-Sensing Research 7, (2016) 127-132.

[8] S.K. Jha, G.S. Joo, G.S. Ra, H.H. Lee, Kim,Y.-S., 2011. Development of PCR microchip for early cancer risk prediction. IEEE Sens. J. 11(9), 2065-2070. 
[9] C.L. Lin, W.H. Chang, C.H. Wang, C.H. Lee, T.Y. Chen, F.J. Jan, G.B. Lee, A microfluidic system integrated with buried optical fibers for detection of Phalaenopsis orchid pathogens, Biosensors and Bioelectronics 63 (2015) 572-579.

[10] X. Zhang, S.B. Lowe, J.J Gooding, Brief review of monitoring methods for loop-mediated isothermal amplification (LAMP), Biosensors and Bioelectronics 61 (2014) 491-499.

[11] T. Notomi, Y. Mori, N. Tomita, H. Kanda, Loop-mediated isothermal amplification (LAMP): principle, features, and future prospects, Journal of microbiology 53(1) (2015) 1-5.

[12] M. Safavieh, M.K. Kanakasabapathy, F. Tarlan, M.U. Ahmed, M. Zourob, W. Asghar, H. Shafiee, Emerging Loop-Mediated Isothermal Amplification-Based Microchip and Microdevice Technologies for Nucleic Acid Detection, ACS Biomater. Sci. Eng. 3 (2016) 278-294.

[13] W. De-Guo, J.D. Brewster, P. Moushumi, P.M. Tomasula, Two methods for increased specificity and sensitivity in Loop-mediated isothermal amplification, Molecules 20 (2015) 6048-6059.D. Lee, M. La Mura, T.R. Allnutt, W. Powell, Detection of genetically modified organisms (GMOs) using isothermal amplification of target DNA sequences, BMC Biotechnology 9 (2009) 7.

[14] G. Gallinella, Parvovirus B19 Achievements and Challenges, ISRN Virology. Article ID 898730, (2013) doi: 10.5402/2013/898730.

[15] S. Rao, G. Pangallo, F. Della Corte, Integrated Amorphous Silicon p-i-n Temperature Sensor for ha formattato: Inglese (Regno Unito) CMOS Photonics. Sensors. 16, (2016) 67.

[16] D. Caputo, G. de Cesare, M. Nardini, A. Nascetti, R. Scipinotti, Monitoring of Temperature Distribution in a Thin Film Heater by an Array of a-Si:H Temperature Sensors, IEEE Sensors Journal 12(5), (2012) 1209-1213.

[17] P.S. Dittrich, A. Manz, Lab-on-a-chip: microfluidics in drug discovery, Nat. Rev. Drug. Discov. 5, (2006) 210-218. 
[18] O. Gandelman, V. Church, C. Moore, G. Kiddle, C. Carne, S. Parmar, H. Jalal, L. Tisi, J. Murray, Novel Bioluminescent Quantitative Detection of Nucleic Acid Amplification in RealTime, PLoS One, 5(11), (2010) e14155.

[19] G. Kiddle, P. Hardinge, N. Buttigieg, O. Gandelman, C. Pereira, C.J. McElgunn, M. Rizzoli, R. Jackson, N. Appleton, C. Moore, L.C. Tisi, J.A. Murray, GMO detection using a bioluminescent real time reporter (BART) of loop mediated isothermal amplification (LAMP) suitable for field use, BMC Biotechnology 12(15), (2012) 1.

[20] C.J. McElgunn, C.R. Pereira, N.J. Parham, J.E. Smythe, M.J. Wigglesworth, A. Smielewska, S.A. Parmar, O.A. Gandelman, N.M. Brown, L.C. Tisi, M.D. Curran, A low complexity rapid molecular method for detection of Clostridium difficile in stool, PloS one 9(1), (2014) e83808.

[21] A. Roda, M. Guardigli, E. Michelini M. Mirasoli, Bioluminescence in analytical chemistry and in vivo imaging, TRAC-Trends in Analytical Chemistry. 28, (2009) 307-322.

[22] M. Seidel, R. Niessner, Chemiluminescence microarrays in analytical chemistry: a critical review. Anal Bioanal Chem. 406(23), (2014) 5589-612.

[23] M. Mirasoli, M. Guardigli, E. Michelini, A. Roda, Recent advancements in chemical luminescence-based lab-on-chip and microfluidic platforms for bioanalysis, J. Pharmaceut. Biomed. 87, (2014) 36-52.

[24] G. de Cesare, A. Nascetti, D. Caputo, Amorphous Silicon p-i-n Structure Acting as Light and Temperature Sensor, Sensors 15(6), (2015) 12260-12272.

[25] D. Caputo, G. de Cesare, C. Manetti, A. Nascetti, R. Scipinotti, Smart thin layer chromatography plate, Lab Chip 7(8) (2007) 978-980. 
[26] P. Novo, V. Chu, J.P. Conde, Integrated fluorescence detection of labeled biomolecules using a prism-like PDMS microfluidic chip and lateral light excitation, Lab on a Chip - Miniaturisation for Chemistry and Biology 14(12), (2014) 1991-1995.

[27] M. Mirasoli, A. Nascetti, D. Caputo, M. Zangheri, R. Scipinotti, L. Cevenini, G. de Cesare, A. Roda, Multiwell cartridge with integrated array of amorphous silicon photosensors for chemiluminescence detection: Development, characterization and comparison with cooled-CCD luminograph, Analytical and Bioanalytical Chemistry 406(23) (2014) 5645-5656.

[28] D. Caputo, G. de Cesare, C. Fanelli, A. Nascetti, A. Ricelli, R. Scipinotti, Amorphous silicon photosensors for detection of ochratoxin a in wine, IEEE Sensors Journal. 12(8), (2012) 2674-2679.

[29] D. Caputo, M. Ceccarelli, G. d Cesare, A. Nascetti, R. Scipinotti, Lab-on-glass system for DNA analysis using thin and thick film technologies, Proceedings of Materials Research Society Symposium, 2009 MRS Spring Meeting 1191 (2009) 53-58.

[30] F. Costantini, A. Nascetti, R. Scipinotti, F. Domenici, S. Sennato, L. Gazza, F. Bordi, N. Pogna, C. Manetti, D. Caputo, G. de Cesare, On-chip detection of multiple serum antibodies against epitopes of celiac disease by an array of amorphous silicon sensors, Rsc Advances 4(4), (2014) 2073-2080.

[31] F. Costantini, C. Sberna, G. Petrucci, C. Manetti, G. de Cesare, A. Nascetti, D. Caputo, Lab-on-chip system combining a microfluidic-ELISA with an array of amorphous silicon photosensors for the detection of celiac disease epitopes, Sensing and Biosensing Res. 6 (2015) 5158.

[32] C.R. Vistas, S.S. Soares, R.M.M. Rodrigues, V. Chu, J.P. Conde, G.N.M. Ferreira, An amorphous silicon photodiode microfluidic chip to detect nanomolar quantities of HIV-1 virion infectivity factor, Analyst 139 (2014) 3709-3713.

[33] G. Bua, E. Manaresi, F. Bonvicini, G. Gallinella, Parvovirus B19 Replication and Expression in Differentiating Erythroid Progenitor Cells, PLoS One 11(2), (2016) e0148547. 
[34] F. Bonvicini, E. Manaresi, G. Bua, S. Venturoli, G. Gallinella, Keeping Pace with Parvovirus B19 Genetic Variability: a Multiplex Genotype-Specific Quantitative PCR Assay, J Clin. Microbiol. 51 (2013) 3753-3759.

[35] T. Notomi, H. Okayama, H. Masubuchi, T. Yonekawa, K. Watanabe, N. Amino, T. Hase, Loop-mediated isothermal amplification of DNA, Nucleic Acids Res. 28(12) (2000) E63.

[36] M. Mirasoli, A. Buragina, L.S. Dolci, M. Guardigli, P. Simoni, A. Montoya, E. Maiolini, S. Girotti, A. Roda, Development of a chemiluminescence-based quantitative lateral flow immunoassay for on-field detection of 2,4,6-trinitrotoluene, Anal. Chim. Acta 721, (2012) 167-172.

[37] A. Nascetti, M. Truglio, P. Valerio, D. Caputo, G. de Cesare, High dynamic range current-todigital readout electronics for lab-on-chip applications, Proceedings of the 4th IEEE International Workshop on Advances in Sensors and Interfaces, IWASI 20116004691 (2011) 77-81.

[38] A. Nascetti, G. Colonia, D. Caputo, G. de Cesare, Sophie: A general purpose sub-picoamps current readout electronics, Lecture Notes in Electrical Engineering 319 (2015) 285-289.

[39] F. Bonvicini, G. Bua, E. Manaresi, G.Gallinella, Antiviral effect of cidofovir on parvovirus B19 replication, Antiviral Research, 113, (2015) 11-18.

[40] G. de Cesare, D. Caputo, M. Tucci, Electrical properties of ITO/crystalline-silicon contact at different deposition temperatures, IEEE Electron Device Letters 33(3), (2012) 327329.

[41] D. Caputo, G. de Cesare, A. Nascetti, R. Scipinotti, a-Si:H temperature sensor integrated in a thin film heater, Phys. Status Solidi A 207(3), (2010) 708-711.

[42] D. Caputo, G. de Cesare, M. Ceccarelli, A. Nascetti, M. Tucci, L. Meda, M. Losurdo, G. Bruno, Characterization of chromium silicide thin layer formed on amorphous silicon films, J. Non-Crystalline Solids, 354, (2008) 2171-2175. 
[43] T.R.E. Simpson, B. Parbhoo, J.L. Keddie, The dependence of the rate of crosslinking in poly(dimethyl siloxane) on the thickness of coatings, Polymer, 44(17), (2003) 4829-4838.

[44] B. Samel, M.K. Chowdhury, G. Stemme, The fabrication of microfluidic structures by means of full-wafer adhesive bonding using a poly(dimethylsiloxane) catalyst, J. Micromech. Microeng. 17 (2007) 1710-1714.

[45] Y. Yamada, M. Itoh, M. Yoshida, Sensitive and rapid diagnosis of human parvovirus B19 infection by loop-mediated isothermal amplification, British Journal of Dermatology 155 (2006) 50-55.

[46] D. Caputo, U. Forghieri, F. Palma, Low-temperature admittance measurement in thin film amorphous silicon structures, Journal of Applied Physics. 82(2), (1997) 733-741.

[47] Lovecchio, N., G. Petrucci, G., Caputo, D., Alameddine, S., Carpentiero, M., Martini, L., Parisi, E., de Cesare, G. Nascetti, A., 2015. Thermal control system based on thin film heaters and amorphous silicon diodes. $6^{\text {th }}$ IEEE International Workshop on Advances in Sensors and Interfaces (IWASI), 277-282.

[48] D. Caputo, G. de Cesare, L.S. Dolci, M. Mirasoli, A. Nascetti, A. Roda, R. Scipinotti, Microfluidic chip with integrated a-Si:H photodiodes for chemiluminescence-based bioassays, IEEE Sensors Journal. 13(7), (2013) 2595-2602.

[49] Y. Fu, H. Zhou, F. Jing, Q. Jin, J. Zhao, G. Li, A microfluidic chip based on surfactant-doped polydimethylsiloxane (PDMS) in a sandwich configuration for low-cost and robust digital PCR. Sensors and Actuators B: Chemical 245 (2017) 414-422. 